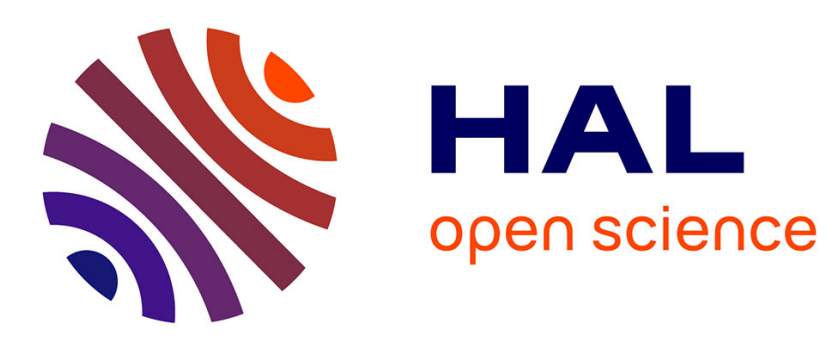

\title{
Time-varying force from dense granular avalanches on a wall
}

\author{
B. Chanut, Thierry Faug, M. Naaim
}

\section{To cite this version:}

B. Chanut, Thierry Faug, M. Naaim. Time-varying force from dense granular avalanches on a wall. Physical Review E : Statistical, Nonlinear, and Soft Matter Physics, 2010, 82 (4), 12 p. 10.1103/PhysRevE.82.041302 . hal-00529731

\section{HAL Id: hal-00529731 \\ https://hal.science/hal-00529731}

Submitted on 26 Oct 2010

HAL is a multi-disciplinary open access archive for the deposit and dissemination of scientific research documents, whether they are published or not. The documents may come from teaching and research institutions in France or abroad, or from public or private research centers.
L'archive ouverte pluridisciplinaire HAL, est destinée au dépôt et à la diffusion de documents scientifiques de niveau recherche, publiés ou non, émanant des établissements d'enseignement et de recherche français ou étrangers, des laboratoires publics ou privés. 
Physical Review E, Volume 82, Issue 4, 041302 (2010)

URL: http://link.aps.org/doi/10.1103/PhysRevE.82.041302

DOI: 10.1103/PhysRevE.82.041302

\title{
Time-varying force from dense granular avalanches on a wall
}

\author{
Benoit Chanut, Thierry Faug* and Mohamed Naaim \\ Cemagref, ETGR, 38402 St. Martin d'Hères, France
}

(Dated: September 2010)

\begin{abstract}
We studied avalanches of cohesionless granular materials down a rough inclined plane and overflowing a wall normal to the incoming flow and to the bottom. This paper focuses on the transient time-varying mean force exerted by the granular stream on the obstacle at various slope inclinations. A nearly triangular dead zone is formed upstream of the obstacle. It largely contributes to the overall force signal at low slope inclinations. It also drives the residual force corresponding to the avalanche tail until its standstill whatever the slope inclination. An analytical hydrodynamic model based on depth-averaged momentum conservation was successfully developed for steady-flow conditions to predict the steady-state force computed from discrete numerical simulations [Faug, Beguin, and Chanut, Phys. Rev. E 80, 021305 (2009)]. The basic equations of the model are briefly reviewed and adapted to transient time-varying flows. The modified hydrodynamic model quite accurately represents the force peak produced by the granular avalanche flows computed from discrete numerical simulations reported in previous studies. A fitting procedure is needed to represent the decrease of the force after the force peak, thus quantifying the different contributions to the mean force on the wall. We show that the weight of each contribution is largely dependent on the slope inclination.
\end{abstract}

*Electronic address: thierry.faug@cemagref.fr 
Physical Review E, Volume 82, Issue 4, 041302 (2010)

URL: http://link.aps.org/doi/10.1103/PhysRevE.82.041302

DOI: 10.1103/PhysRevE.82.041302

\section{Contents}

1. Introduction

2. Hydrodynamic model 4

2.1. Equations for steady flows 4

2.2. Equations for avalanches 6

3. Model predictions compared to discrete numerical simulations results 8

3.1. Discrete numerical simulations 8

Simulation method, contact law and microscopic parameters 8

Simulated systems and measures $\quad 8$

3.2. Measured and predicted dead zone lengths 9

3.3. Measured and predicted forces 10

Influence of the basal friction $\mu_{z m}$ : a time-varying value? 11

Influence of the time-derivative term in momentum balance 12

Influence of $L, k, \kappa$ and $\beta \quad 13$

Influence of $\mu_{z m}$ : distinguishing flows below and above $\theta_{\max }$ ? 14

3.4. Various contributions to the total force $\quad 15$

4. Conclusion 16

$\begin{array}{lr}\text { 5. Acknowlegments } & 18\end{array}$

$\begin{array}{ll}\text { References } & 18\end{array}$

\section{INTRODUCTION}

Granular flows around obstacles and the force that granular flows are able to exert on the obstacle are of major importance in several applications such as storage and conveying of bulk solids in industrial processes [1] as well as protection dams against geophysical flows [2-6]. Granular drag on objects was approached by the pioneering work of Wieghardt $[7,8]$ and was followed by several studies in a relatively quasi-static granular regime on the one hand [9-14] and in the rapid-dilute-granular regime on the other hand [15-18] including 
Physical Review E, Volume 82, Issue 4, 041302 (2010)

URL: http://link.aps.org/doi/10.1103/PhysRevE.82.041302

DOI: 10.1103/PhysRevE.82.041302

interstitial gas effects $[19,20]$. Many studies on granular flows around obstacles focused on the shock waves occurring in front of the obstacles in the rapid regime $[17,18,21-26]$. To date, granular drag in the dense regime has received little attention, referring to the so-called granular liquid regime mentioned in [27].

A condition to obtain such a dense regime is a rough bottom (typical size roughness close to the diameter of the moving grains). In presence of obstacles, this complex dense regime leads to the formation of large stagnant zones or "dead zones" [28, 29] in front of the obstruction. The following conditions are needed for dead zone formation: (i) an obstacle of typical size close to the incoming flow thickness, and (ii) a relatively low Froude number of the incident flow ( $F r \sim 1$ typically). When these dead zones occur, the grains that are not trapped inside the dead zone continue to flow over the obstacle in the absence of what is called a granular jump, i.e., a large discontinuity in flow and velocity. These granular jumps have been outlined earlier for rapid flows on relatively smooth beds [30-33]. As shown for freesurface gravity-driven granular flows overflowing a wall in the steady dense regime, a nearly triangular dead zone, whose length depends on the slope inclination, is formed upstream of the obstacle [see experimental evidence in 28]. This dead zone largely influences the force on the wall, as shown by discrete numerical simulations in steady flow conditions [29]. In this steady dense regime, a simple hydrodynamic analytical model has been proposed and successfully tested to reproduce the mean force on the wall computed from discrete numerical simulations [29]. In this paper, we propose to slightly modify the hydrodynamic model in order to adapt it to transient, time-varying granular avalanches. This corresponds to the situation typically encountered in geophysical flows when avalanches flow over protection dams in the run-out zone [5].

The first section reviews the hydrodynamic model's equations for the steady regime [see details in 29] and describes the assumptions made to establish the time-varying model equations. In particular, exponential growth over time is postulated for the dead zone length. In the second step, the exponential equation describing the dead zone length is successfully validated on discrete numerical simulations briefly described in the present paper (and previously reported in [34]). The predicted force is then directly cross-compared to the mean force derived from the discrete numerical simulations with a careful focus on the model's sensitivity to each of the various free parameters. The model's predictions are in good agreement with the numerical results to predict the force at short and intermediate times. 
Physical Review E, Volume 82, Issue 4, 041302 (2010)

URL: http://link.aps.org/doi/10.1103/PhysRevE.82.041302

DOI: 10.1103/PhysRevE.82.041302

In particular, the maximum force is accurately predicted by the hydrodynamic model. For slopes greater than a critical angle $\theta_{\max }$, we show that a calibration of the friction force acting at the base of the dead zone is needed to be able to catch the numerical results with the hydrodynamic model at larger times when the avalanche comes to a standstill. $\theta_{\max }$ is the angle above which steady recirculating flows do not remain uniform [29, 35-37]. The resulting basal friction force was found to vanish when the slope inclination is greater than the angle $\theta_{\max }$. The various contributions to the force derived from the model are also quantified. A discussion on the usefulness of this avalanche force model within the framework of depth-averaged equations, recently revisited for dense granular avalanches [38], is finally given, and the possible model extension to more complex obstacle geometries is suggested.

\section{HYDRODYNAMIC MODEL}

\subsection{Equations for steady flows}

We consider a steady granular stream down a rough inclined slope $\theta$ characterized by its flow depth $h$, its depth-averaged velocity $\bar{u}=\frac{1}{h} \int_{0}^{h} u(y) d y$ and its depth-averaged fluid density $\bar{\rho}=\bar{\phi} \rho_{P} . \bar{\phi}$, respectively $\rho_{P}$, are the depth-averaged volume fraction and the particle density of the granular material. The granular stream overflows an obstacle of height $H$, as shown in Fig. 1(a). We define $\mathbf{x}$, respectively $\mathbf{y}$, as the unit vectors parallel, respectively normal, to the bottom [Fig. 1(a)]. Based on momentum conservation applied to the control volume $V_{0}$ defined in Fig. 1(a), the hydrodynamic model proposed and described in detail in [29] makes it possible to define the normal force $F_{n}$ exerted on a wall by a steady dense granular flow as the sum of a dynamic force $F_{d}$, a hydrostatic force $F_{p}$, and a force based on the weight $F_{w}$ of the control volume minus the basal friction $F_{f}$ with the rough bottom:

$$
\begin{aligned}
F_{n} & =F_{d}+F_{p}+F_{w}-F_{f}, \\
F_{d} & =\beta \bar{\rho} \bar{u}^{2} h\left[1-\left(1-\kappa \alpha^{0}\right) \cos \alpha^{0}\right], \\
F_{p} & =\frac{1}{2} k \bar{\rho} g h^{2} \cos \theta \\
F_{w}-F_{f} & =\left[\bar{\rho}\left(V_{0}-\frac{1}{2} L H\right)+\frac{1}{2} \rho_{z m} L H\right] g\left(\sin \theta-\mu_{z m} \cos \theta\right),
\end{aligned}
$$

where the $\beta$ factor is related to the velocity profile and is defined by $\frac{1}{h} \int_{0}^{h} u^{2}(y) d y=\beta \bar{u}^{2}$, 
Physical Review E, Volume 82, Issue 4, 041302 (2010)

URL: http://link.aps.org/doi/10.1103/PhysRevE.82.041302

DOI: 10.1103/PhysRevE.82.041302

$\kappa$ is a velocity reduction coefficient equal to $(1-e) /(\pi / 2)$ with $e$ the restitution coefficient [29], and $k$ is the earth pressure coefficient classically introduced for granular flows [38, 39] representing the ratio of the longitudinal normal stress $\sigma_{x x}$ to the vertical normal stress $\sigma_{y y}$. $\rho_{z m}$ is the mean density inside the dead zone and should be greater than the density $\bar{\rho}$ due to the expected compression of the material trapped upstream of the wall.

To close the model, the following additional equations are needed to define the deflecting angle $\alpha^{0}$, the dead zone angle $\alpha_{z m}^{0}$, the free-surface angle $\alpha_{s l}^{0}$, the control volume $V_{0}$ (including the upstream fluid volume disturbed by the obstacle), the length of the influence zone $L$ upstream of the obstacle, and the basal friction coefficient $\mu_{z m}$ :

$$
\begin{array}{r}
\alpha^{0}=\frac{1}{2}\left(\alpha_{z m}^{0}+\alpha_{s l}^{0}\right), \\
\alpha_{z m}^{0}=\theta-\theta_{\min }, \\
\alpha_{s l}^{0}\left(\theta<\theta_{\max }\right)=\frac{\theta_{\min }}{\theta_{\max }-\theta_{\min }}\left(\theta-\theta_{\min }\right), \\
\alpha_{s l}^{0}\left(\theta>\theta_{\max }\right)=\frac{\pi}{2}-\left(\frac{\theta_{\min }-\pi / 2}{\theta_{\max }-\pi / 2}\right)(\pi / 2-\theta), \\
V_{0}=\frac{1}{2} h L\left[2+\tan \left(2 \alpha^{0}-\arctan \frac{H}{L}\right)\left(\frac{L}{h}-\delta_{h} \sin \alpha^{0}\right)+\frac{\delta_{h}}{L}(H-h) \sin \alpha^{0}\right], \\
L=\frac{H}{\tan \left(\theta-\theta_{\min }\right)}, \\
\mu_{z m}=\tan \theta_{\min } .
\end{array}
$$

The angle $\alpha_{z m}^{0}$ is the angle of the nearly triangular dead zone formed upstream of the wall. The angle $\alpha_{s l}^{0}$ denotes the angle of the upstream free surface disturbed by the presence of the obstacle. The angle $\alpha^{0}$ is the resulting mean angle between the bottom and the $\mathbf{u}^{*}$ direction, where $\mathbf{u}^{*}$ is the outgoing flow velocity at section $\left(S^{*}\right)$ defined in Fig. 1(a). All these variables can be seen in Fig. 1(a). The angles $\theta_{\min }$ and $\theta_{\max }$ define the slope inclination range for which steady and uniform granular flows are possible [29, 35-37] if a constant input discharge is supplied. Assuming that $\delta_{h}$ is simply inversely proportional to the velocity reduction $\delta_{u}=\bar{u}^{*} / \bar{u}=1-\kappa \alpha[29], \delta_{h}$ is defined by $\delta_{h}=h^{*} / h=1 /(1-\kappa \alpha)$, where $h^{*}$ is the thickness of the outgoing flow at section $\left(S^{*}\right)$ defined in Fig. 1(a). The entire calculation to derive the system of Eqs. (1) and (2) is detailed in [29].

The discrete numerical simulations on the steady flows presented in [29] show that the dead zone angle with the horizontal, $\theta-\alpha_{z m}^{0}$ [see Fig. 1(a)], was found to be constant and 
Physical Review E, Volume 82, Issue 4, 041302 (2010)

URL: http://link.aps.org/doi/10.1103/PhysRevE.82.041302

DOI: 10.1103/PhysRevE.82.041302

equal to $\theta_{\min }$ for any slope, which gives Eq. (2b). Therefore, it was possible to derive the length of the influence zone $L$ according to Eq. (2f). It was also found that the basal friction coefficient $\mu_{z m}$ was constant and equal to $\tan \theta_{\text {min }}$, which gives Eq. (2g). Further details can be found in [29]. These results must be modified for the granular avalanches investigated in this paper, which are time-varying phenomena. This will be discussed in the following section.

\subsection{Equations for avalanches}

In the case of finite volume granular avalanches, we have to consider the additional term $\frac{\mathrm{d}}{\mathrm{d} t}\left(\iint_{V} \rho \mathbf{u} d V\right)$ in the momentum balance (per unit width). This term must be taken into account beside Eqs. (1b), (1c), and (1d) provided the time-varying values of the flow depth $h(t)$, the depth-averaged velocity $\bar{u}(t)$, and the depth-averaged density $\bar{\rho}(t)$. The time $t=0$ corresponds to the avalanche release. The average velocity inside the dead zone is nil, which leads to $\frac{\mathrm{d}}{\mathrm{d} t}\left(\iint_{V} \rho \mathbf{u} d V\right)=\frac{\mathrm{d}}{\mathrm{d} t}\left[\iint_{V_{0}-\frac{1}{2} H L} \rho \mathbf{u} d V\right]$. Let us consider the approximation $\frac{\mathrm{d} M}{\mathrm{~d} t}=$ $\frac{\mathrm{d}}{\mathrm{d} t}\left(\iint_{V} \rho \mathbf{u} d V\right) \cdot \mathbf{x} \simeq \frac{\mathrm{d}}{\mathrm{d} t}\left[\rho_{V} u_{V}\left(V_{0}-\frac{1}{2} H L\right)\right]$, where $u_{V}$ and $\rho_{V}$ are the mean velocity and density inside the volume $V_{0}-\frac{1}{2} H L$. Then, neglecting the term $\frac{\mathrm{d} M}{\mathrm{~d} t}$ is a reasonable assumption if the variations of the momentum $\frac{\mathrm{d}}{\mathrm{d} t}\left(\rho_{V} u_{V}\right)$ and of the volume $\frac{\mathrm{d}}{\mathrm{d} t}\left(V_{0}-\frac{1}{2} H L\right)$ are small. This is almost true when the avalanche decelerates before coming to a standstill (avalanche tail after the peak pressure), but this assumption may fail at short and intermediate times when the avalanche front impacts the obstacle, with possibly large time-velocity, flow depth, and pressure gradients. We will come back to this assumption in Sec. 3.3.

We call $\alpha_{z m}$ the dead zone angle, $\alpha_{s l}$ the free-surface angle, and $\alpha$ the deflection angle which are time-varying in the avalanche regime considered here. Only at the first impact of the avalanche front with the wall does the influence zone length remain at zero (the incident flow is still undisturbed), corresponding to a limit value of the angles $\alpha_{z m}$ and $\alpha_{s l}$ equal to $\pi / 2$. If the avalanche volume is large enough, we can expect to reach a steady state similar to the one investigated in [29]. This steady regime may lead to the angle value corresponding to the steady regime defined by Eqs. (2b), (2c), and (2d). At this stage, and without accurate experimental knowledge of these angles' changes over time, we can expect to reproduce this behavior by exponential laws in the following form as soon as the avalanche has reached the wall: 


$$
\begin{aligned}
\alpha_{z m}(t) & =\alpha_{z m}^{0}+\left(\frac{\pi}{2}-\alpha_{z m}^{0}\right) e^{\left(T_{i}-t\right) / \tau} \\
\alpha_{s l}(t) & =\alpha_{s l}^{0}+\left(\frac{\pi}{2}-\alpha_{s l}^{0}\right) e^{\left(T_{i}-t\right) / \tau}
\end{aligned}
$$

where $\alpha_{z m}^{0}$ and $\alpha_{s l}^{0}$ are the values in the steady state [Eqs. (2b), (2c) and (2d)] and $\tau$ is a characteristic time. The time $T_{i}$ corresponds to the impact of the avalanche with the wall. Eqs. (3a) and (3b) are valid if $t \geq T_{i} . \alpha_{z m}(t)$ and $\alpha_{s l}(t)$ are equal to $\pi / 2$ when $t<T_{i}$. It is reasonable to assume that $\tau$ is similar for $\alpha_{z m}$ and $\alpha_{s l}$, since the latter angle is directly influenced by the former angle. The value of $\tau$ will be discussed in the next section. The time-varying deflecting angle $\alpha(t)$ is simply derived from Eq. (2a), which remains valid if the above time-varying angles $\alpha_{z m}(t)$ and $\alpha_{s l}(t)$ are considered. The limit values of the angles established for the steady regime may be inappropriate to describe the final state when the avalanche comes to a standstill. This point will be discussed in Sec. 3.2. The time-varying influence zone length $L(t)$ is then defined as:

$$
L(t)=\frac{H}{\tan \left[\alpha_{z m}(t)\right]},
$$

and the control volume $V_{0}$ is time-varying and defined by Eq. (2e) provided the appropriate time-varying values of $L(t)[$ Eq. (4)] and $\alpha(t)$.

In steady regime [29], the basal friction coefficient $\mu_{z m}$ was found constant for any slope and equal to $\tan \theta_{\min }$ on the one hand and the dead zone angle with the horizontal, $\theta-\alpha_{z m}^{0}$, was also found constant for any slope and equal to $\theta_{\min }$ on the other hand. This results leads to the following equation in steady regime: $\mu_{z m}=\tan \left(\theta-\alpha_{z m}^{0}\right)$. This last equation suggests a simple geometrical relation between the basal friction coefficient and the dead zone angle with the horizontal. If such a statement holds for the time-varying avalanche, the basal friction coefficient should be time-varying and is likely to be defined by the following equation with respect to the dead zone geometry:

$$
\mu_{z m}(t)=\tan \left[\theta-\alpha_{z m}(t)\right]
$$

Whether the above time-varying value of $\mu_{z m}$ from Eq. (5) should be chosen instead of a constant value equal to $\tan \theta_{\min }$ will be discussed in Sec. 3.3. It should be noted that the basal friction coefficient defined here is used to describe a time-averaged macroscopic 
Physical Review E, Volume 82, Issue 4, 041302 (2010)

URL: http://link.aps.org/doi/10.1103/PhysRevE.82.041302

DOI: 10.1103/PhysRevE.82.041302

behavior without going into the details of the complex physical processes acting inside the dead zone. The fluctuating force chain network observed inside the dead zone and leading to high-frequency force fluctuations (Figure 4(a) in [29] and Figure 4 in [34]) is beyond the scope of the depth-averaged hydrodynamic model presented in this paper.

\section{MODEL PREDICTIONS COMPARED TO DISCRETE NUMERICAL SIMU- LATIONS RESULTS}

\subsection{Discrete numerical simulations}

\section{Simulation method, contact law and microscopic parameters}

Numerical simulations were carried out using the molecular-dynamics method as introduced by Cundall [40] and successfully used to simulate dense granular flows [see, for example 36, 41, 42]. The method assumes rigid spheres interacting through a damped linear spring for the normal force and a linear spring restricted to a threshold value according to friction between grains described by a Coulomb condition for the tangential force. Four microscopic parameters are needed for the model: the normal stiffness $k_{n}\left(=10^{4} \mathrm{~N} \mathrm{~m}^{-1}\right.$ in our simulations), the tangential stiffness $k_{t}\left(=1 / 2 k_{n}\right)$, the local particle friction $\mu(=0.5)$, and the damping coefficient related to the restitution coefficient $e(=0.5)$. Further details on the numerical method and the choice of the values given to the microscopic parameters are presented in [29, 34] and references therein.

\section{Simulated systems and measures}

The simulated systems consisted of an inclined slope and an upstream reservoir of length $L_{r}=300 d$, as shown in Fig. 1(b). The grains in motion were spheres with a slight polydispersity $( \pm 10 \%$ in size). The simulated system worked as a purely $2 \mathrm{D}$ system made of disks but with spheres whose centers were forced to stay in the 2D plane $(x, y)$ defined in Fig. 1(b). Because of the thickness of the spheres in the third dimension $z$ [see Fig. 1(b)], we will consider 3D volume fractions (see Sec. 3.3). The mean grain diameter was $d=1 \mathrm{~mm}$ and the particle density was $\rho_{P}=2450 \mathrm{~kg} \mathrm{~m}^{-3}$ corresponding to glass material. The roughness of the bottom was made with grains measuring $d$ in diameter with the same properties as the 
Physical Review E, Volume 82, Issue 4, 041302 (2010)

URL: http://link.aps.org/doi/10.1103/PhysRevE.82.041302

DOI: 10.1103/PhysRevE.82.041302

grains in motion. A constant volume was initially released from the reservoir by an aperture of constant height $H_{r}=35 \mathrm{~d}$. The results of the numerical tests are presented in greater detail elsewhere [34]. The granular avalanches considered here differ from the stationary flows generated from a recirculation system investigated in [29]. The avalanche flows are more complex because they correspond to transient, time-varying granular flows.

First, we investigated flows with no obstacle in a large range of slope inclinations $\left(16^{\circ} \leq\right.$ $\left.\theta \leq 32^{\circ}\right)$ and we characterized the changes over time in flow depth $h$, depth-averaged velocity $\bar{u}$ and volume fraction $\bar{\phi}$ at various locations, $x / d$, from the reservoir. The typical changes over time in flow depth and velocity are given in Fig. 2 for $\theta=24^{\circ}$ at the position $x_{0} / d=500$. Second, we measured the force over time exerted by the granular avalanche on an obstacle located at the position $x_{0} / d=500$. The obstacle is a wall of height $H$ normal to the incoming flow and normal to the bottom. The flow depth over time at location $x_{0} / d=500$ shows a maximum (see example in Fig. 2). $H$ was systematically set to be equal to this maximum value of the flow depth at the location $x_{0} / d=500$. High-frequency force fluctuations were observed, as shown in [34, Fig. 4]. In this paper, we focus on the time-averaged normal force $F_{n}$ only. Typical forces evolving with time are depicted in Fig. 3. The force-vs-time signals are characterized by three phases: (i) a short-duration force increase, (ii) a force peak whose width increases with decreasing slope inclination, (iii) a long-lasting force decrease up to a residual force whose value decreases with decreasing slope inclination. Further details and discussion of the numerical results can be found in [34]. Further results are also given in Sec. 3.3 when these numerical results and the hydrodynamic model prediction are cross-compared.

\subsection{Measured and predicted dead zone lengths}

Importantly, the dead zone length determines the magnitude of the control volume $V_{0}$ and therefore the forces $F_{w}$ and $F_{f}$ in Eqs. (1a) and (1d). The empirical laws proposed to estimate the change over time of the dead zone angle [Eq. (3a)] and the influence zone length [Eq. (4)] were directly compared to the results of the numerical simulations. This helped the model calibration before focusing on the mean force exerted on the wall. The so-called dead zone was defined as the region inside which the grains had an individual velocity $v$ smaller than a threshold velocity $v_{t}$. For steady recirculating flows investigated in [29], $v_{t}$ was chosen 
Physical Review E, Volume 82, Issue 4, 041302 (2010)

URL: http://link.aps.org/doi/10.1103/PhysRevE.82.041302

DOI: 10.1103/PhysRevE.82.041302

equal to $0.05 \bar{u}$, with $\bar{u}$ the steady averaged velocity of the control flows with no obstacle. $v_{t}$ was slope-dependent. In the avalanche regime here, the depth-averaged velocity $\bar{u}$ was not only slope-dependent but also time-varying. For the sake of simplicity, we used a constant threshold velocity equal to $510^{-2} \mathrm{~m} \mathrm{~s}^{-1}$ whatever the slope and the time $t$. Furthermore, the dead zone shape was calculated at a frequency of $10 \mathrm{~Hz}$. This allowed us to roughly estimate the average shape of the dead zone without consuming too much calculation time. Figure 4 gives a typical example of the changes over time of the dead zone shape. This is shown to be nearly triangular in spite of the observed fluctuations in the dead zone profiles (choosing a time-varying threshold velocity $v_{t}$ also depending on the slope inclination and increasing the acquisition frequency would have smoothed the results). Figure 5 displays the change over time of the dead zone length for the following slopes: $\theta=16^{\circ}, 24^{\circ}$ and $30^{\circ}$. The results for other slopes are very similar. We also reported the prediction from Eq. (4). The comparison shows that the numerical data are relatively well reproduced at short and intermediate times for any slope provided a value of $\tau=0.40 \mathrm{~s}$. At longer times, the predictions remain satisfactory even if the dead zone length tends to increase slightly at longer times, whereas the exponential law predicts a saturation to the value corresponding to the steady regime. It may lead to a slight underestimation of the dead zone length by the proposed empirical law at large times.

\subsection{Measured and predicted forces}

In this section is studied the model's sensitivity to (i) the basal friction coefficient $\mu_{z m}$, (ii) the time-derivative term in momentum balance, (iii) the dead zone length and the model parameters $\beta, \kappa$ and $k$. First, we show that a time-varying value of the basal friction is more efficient than a constant value derived from the steady regime to catch the force peak. However, a gap between numerical results and model prediction exists at large times after the force peak and this gap increases with increasing slope. Second, we show that taking into account or not the time-derivative term in momentum balance has little effect on the results. Third, the sensitivity of the model prediction to the dead zone length and to the model parameters $\beta, \kappa$ and $k$ is investigated. It is shown that varying $L, \beta, \kappa$ or $k$ does not improve the model predictions at large times (avalanche tail). Finally, we show that it is needed to fit the basal friction in order to be able to catch the force not only at short and 
Physical Review E, Volume 82, Issue 4, 041302 (2010)

URL: http://link.aps.org/doi/10.1103/PhysRevE.82.041302

DOI: 10.1103/PhysRevE.82.041302

intermediate times (close to the force peak) but also at large times (avalanche tail). The various steps of the sensitivity study are detailed below.

\section{Influence of the basal friction $\mu_{z m}$ : a time-varying value?}

Figure 6 gives the hydrodynamic model predictions compared to the numerical data using the following set of the model's free parameters: $\theta_{\min }=14^{\circ}, \theta_{\max }=24^{\circ}$ (both angle values were previously determined in $[29,34]$ and are compatible with previous studies with the same granular material in 2D geometry [37, 43]), $k=1, \kappa=0.32(e=0.5)$ and $\beta=1$. Investigating in detail the possible influence of the slope at different times on the density of the dead zone was beyond the scope of our study. We simply assume here that the mean density of the dead zone is close to the random close packing: $\rho_{z m} \simeq \rho_{\max }=\phi_{\max } \rho_{P}$ where $\phi_{\max }=0.64$ is the random close packing for a $3 \mathrm{D}$ system [44]. We use here $\phi_{\max }=0.64$ (real 3D system) instead of $\phi_{\max }=0.82$ (real 2D system) in order to be consistent with the computed forces which depend on the density of the system made of 3D spherical particles. In Fig. 6 are presented the results for the following slopes: $\theta=18^{\circ}, 22^{\circ}$ and $30^{\circ}$. The results for other slopes are similar.

If we consider a time-varying basal friction coefficient, i.e., $\mu_{z m}(t)=\tan \left[\theta-\alpha_{z m}(t)\right]$, the predictions are shown to be relatively good at short and intermediate times for most of the slope inclinations. The model predictions given in Fig. 6 are drawn without including the term $\frac{\mathrm{d} M}{\mathrm{~d} t}$ in momentum balance. This result tends to show that not considering this term may be a reasonable assumption for the avalanche flows investigated here. In the following section are discussed the model predictions including an approximation of this time-derivative term. On the other hand, the force is generally underestimated at higher times (after the force peak). We will come back to this discrepancy between model predictions and numerical data later in the paper. Figure 6 also shows the model's predictions with $\mu_{z m}=\tan \left(\theta_{\min }\right)$, which give less satisfactory results to reproduce the force at short and intermediate times.

The maximum force, or force peak, exerted by the granular avalanche on the wall must be considered. Figure 7 shows the maximum force $F_{\max }$ normalized by a typical force $F_{0}$ versus the slope inclination where $F_{0}$ is defined as: (i) the typical dynamic force of the control flow with no obstacle, $F_{0}^{d y n}=1 / 2 \bar{\rho}_{m} \bar{u}_{m}^{2} h_{\max }$ (where $h_{\max }$ is the maximum height, $\bar{u}_{m}$ and $\bar{\rho}_{m}$ are the corresponding depth-averaged velocity and fluid density, taken at same time $t$ and 
Physical Review E, Volume 82, Issue 4, 041302 (2010)

URL: http://link.aps.org/doi/10.1103/PhysRevE.82.041302

DOI: 10.1103/PhysRevE.82.041302

same position $x_{0} / d=500$ ) and (ii) the typical hydrostatic force of the control flow with no obstacle, $F_{0}^{\text {hydro }}=1 / 2 \bar{\rho}_{m} g h_{\text {max }}^{2} \cos \theta$. Model predictions to reproduce the numerical values of the ratios $F_{\max } / F_{0}^{d y n}$ and $F_{\max } / F_{0}^{\text {hydro }}$ are better with $\mu_{z m}(t)=\tan \left[\theta-\alpha_{z m}(t)\right]$ than with $\mu_{z m}=\tan \theta_{\min }$. In the following, we will systematically keep the time-varying value $\mu_{z m}(t)=\tan \left[\theta-\alpha_{z m}(t)\right]$ with respect to the changes over time in the dead zone angle.

The numerical results from Fig. 7 show that above $\theta_{\max }$ the maximum force is accurately described by $F_{\max } \simeq C_{u} F_{0}^{d y n}$ (with $C_{u}=1$ ) while below $\theta_{\max }$, it is clearly described by $F_{\text {max }} \simeq C_{h} F_{0}^{\text {hydro }}$ (with $C_{h} \simeq 6$ ). Above $\theta_{\max }$, the maximum force scales as $F_{\max } \propto u_{m}{ }^{2} h_{\max }$ (dynamic force). Below $\theta_{\text {max }}$, the maximum force scales as $F \propto h_{\text {max }}^{2}$ (hydrostatic force), but, because of the influence of the dead zone, a force equal to around six times the incoming hydrostatic force is mobilized. The hydrodynamic model is able to reproduce these two regimes remarkably well. It should be noted that a similar behavior has also been evidenced for steady-flow conditions [see Figure 8(b) in 29].

\section{Influence of the time-derivative term in momentum balance}

As previously discussed, the temporal variation of the momentum $\frac{\mathrm{d} M}{\mathrm{~d} t}$ can be approximated with the following equation:

$$
\frac{\mathrm{d} M}{\mathrm{~d} t}=\frac{\mathrm{d}}{\mathrm{d} t}\left(\iint_{V} \rho \mathbf{u} d V\right) \cdot \mathbf{x} \simeq \frac{\mathrm{d}}{\mathrm{d} t}\left[\rho_{V} u_{V}\left(V_{0}-\frac{1}{2} H L\right)\right]
$$

This term can be estimated if we consider the following assumptions: (i) $\rho_{V} \simeq \bar{\rho}$ (the overall density inside $V_{0}-\frac{1}{2} H L$ is assumed equal to the depth-averaged density of the incoming flow) and (ii) $u_{V}=\frac{1}{2} \bar{u}\left(1+\delta_{u}\right.$ ) (the overall velocity inside $V_{0}-\frac{1}{2} H L$ is close to the mean value given by $\bar{u}$ and $\left.\bar{u}^{*}\right)$. Figure 8 shows the comparison between the hydrodynamic model predictions including the term $\frac{\mathrm{d} M}{\mathrm{~d} t}$ or not for the following slopes: $\theta=18^{\circ}, 24^{\circ}$ and $30^{\circ}$. The results for other slopes are very similar. The influence of this term remains weak. However, we can detect a better accuracy of the model to describe the force signal at small times, corresponding to the short-duration increase of the force before reaching the maximum value. Figure 9 displays the maximum force $F_{\max }$ normalized by the typical forces $F_{0}$ (dynamic and hydrostatic) versus the slope inclination. Model predictions including the approximated value of $\frac{\mathrm{d} M}{\mathrm{~d} t}$ or not are displayed. The model predictions on maximum force are slightly influenced by the time-derivative term in momentum balance. 
Physical Review E, Volume 82, Issue 4, 041302 (2010)

URL: http://link.aps.org/doi/10.1103/PhysRevE.82.041302

DOI: 10.1103/PhysRevE.82.041302

Influence of $L, k, \kappa$ and $\beta$

As previously mentioned and shown in Fig. 5, the value of the dead zone length $L$ is shown to be well predicted by the exponential law. However, it may be noticed a slight underestimate of the dead zone length at long times in Fig. 5. Underestimating $L$ leads to underestimating $V_{0}$, resulting in a lower force $F_{w}$. Hence, increasing $L$ can reduce the gap between the model predictions and the numerical data. However, we did not find reasonable $L$ values which allowed us to match the numerical data for any slope and time. As an example, it is needed to multiply the dead zone length by a factor 1.5 to catch the measured force at large times for $\theta=22^{\circ}$. The resulting dead zone length is then far from the measured one and the good prediction at earlier times, before and at the force peak, is completely lost (the force peak being largely overestimated). It can be concluded that the observed discrepancies between the hydrodynamic model predictions and the discrete numerical results at large times after the force peak are not explained by an error in estimating $L$ with the proposed exponential law.

Figure 10 gives the hydrodynamic model predictions compared to the numerical data using various sets of the model's free parameters $k, \kappa(e)$, and $\beta$. Four triplets are considered: (i) $k=1, \kappa=0.32(e=0.5)$, and $\beta=1$; (ii) $k=1.12, \kappa=0.32(e=0.5)$, and $\beta=1$; (iii) $k=1, \kappa=0.32(e=0.5)$, and $\beta=5 / 4$; and (iv) $k=1, \kappa=0.13(e=0.8)$, and $\beta=1$. The value $\beta=1$ corresponds to the exact value for plug flows [39], whereas $\beta=5 / 4$ refers to a Bagnold-like velocity profile [45]. The value $k=1$ corresponds to isotropic material conditions whereas $k=1.12$ is derived from a Mohr-Coulomb plasticity prediction [39] when the basal and the internal friction coefficients are taken equal to $\tan \theta_{\min }$ [46]. In Fig. 10 are presented the results for three slope inclinations: $\theta=16^{\circ}, 24^{\circ}$, and $32^{\circ}$. We found identical trends for the slopes not depicted in Fig. 10.

The model's sensitivity to $\beta$ and $\kappa$ is insignificant at low slopes whatever the time. The model's sensitivity increases with increasing slope inclination but only at short and intermediate times (close to the force peak). Varying $\beta$ and $\kappa$ does not change the model predictions at larger times (avalanche tail). The model's sensitivity to $k$ is generally insignificant for any slope whatever the time. It can be concluded that changing the values of $k, \kappa$ (or $e$ ), and $\beta$ does not explain the observed discrepancies between the hydrodynamic model predictions and the discrete numerical results at large times after the force peak. 
Physical Review E, Volume 82, Issue 4, 041302 (2010)

URL: http://link.aps.org/doi/10.1103/PhysRevE.82.041302

DOI: 10.1103/PhysRevE.82.041302

\section{Influence of $\mu_{z m}$ : distinguishing flows below and above $\theta_{\max }$ ?}

In this section is investigated the influence of the basal friction $\mu_{z m}$ in greater detail. At this stage, and for a lack of better knowledge on the frictional processes involved at the base of the dead zone, we suggest using the basal friction coefficient $\mu_{z m}$ as a calibration parameter. We simply assume that the real basal friction coefficient named $\mu_{z m}^{*}$ is proportional to the basal friction coefficient defined by Eq. (5) where the proportionality coefficient $c_{\mu}$ is a calibration parameter:

$$
\mu_{z m}^{*}(t)=c_{\mu} \tan \left[\theta-\alpha_{z m}(t)\right]
$$

Figure 11 shows the results of the model's calibration on the numerical data for the following slopes: $\theta=18^{\circ}, 22^{\circ}$ and $28^{\circ}$. The results for other slopes are similar. The bestfitting curves have been obtained by a simple method of least squares. The hydrodynamic model can match the numerical data well after the force peak if the following values for $c_{\mu}$ depending on the slope inclination are considered: $c_{\mu}=0.99$ for $\theta=16^{\circ}, c_{\mu}=0.99$ for $\theta=18^{\circ}, c_{\mu}=0.86$ for $20^{\circ}, c_{\mu}=0.52$ for $22^{\circ}, c_{\mu}=0.20$ for $\theta=24^{\circ}$, and $c_{\mu}=0$ for $\theta=26^{\circ}, 28^{\circ}, 30^{\circ}$, and $32^{\circ}$.

Importantly the angle $\theta_{\max }$ that defines a critical angle above which no steady and uniform flows are possible even if a constant input discharge in granular materials is supplied. This angle $\theta_{\max }$ is determined by measuring the thickness of the granular material left by the granular stream and depends on the flowing grains and roughness properties (see [35, 37] and references therein). $\theta_{\max }$ was estimated at $24^{\circ}$ for the material studied $[29,34]$, which is compatible with previous studies on similar 2D granular systems [37, 43]. Physically, $\theta_{\max }$ can be interpreted as the critical friction angle above which grains cannot be trapped in the bottom roughness. Thus, one can expect different friction processes at the base of the dead zone if the slope inclination is below or above $\theta_{\max }$. Figure 12 depicts the calibration coefficient $c_{\mu}$ versus the slope inclination. The curve shows a change in behavior, which, remarkably, acts around the critical angle $\theta_{\max }$. Above $\theta_{\max }, c_{\mu}$ vanishes, indicating that the friction force at the base of the dead zone is nil. Below $\theta_{\max }, c_{\mu}$ sharply increases and tends to reach a value equal to 1 when the slope inclination tends toward $\theta_{\text {min }}$.

At this stage, we are not able to explain this peculiar result derived from the fitting process and showing a transition around $\theta=\theta_{\max }$. We can only suggest the idea or the 
Physical Review E, Volume 82, Issue 4, 041302 (2010)

URL: http://link.aps.org/doi/10.1103/PhysRevE.82.041302

DOI: 10.1103/PhysRevE.82.041302

intuition that this behavior is related to the function $h_{\text {stop }}(\theta)$ previously evidenced from numerical and experimental simulations [27, 35, 37]. Indeed let us imagine a situation for which the wall would be removed. Above $\theta_{\max }$, all the grains stored upstream of the wall will flow downward obeying $h_{\text {stop }}\left(\theta>\theta_{\max }\right)=0$ and suggesting that the average basal friction (resulting from grain scale effects such as trapping) is nil. Below $\theta_{\text {max }}$, the grains will form a deposit obeying $h_{\text {stop }}\left(\theta<\theta_{\max }\right)>0$ suggesting that the average basal friction is non-zero. The thickness of this deposit will increase with decreasing slope inclination (increase of $h_{\text {stop }}(\theta)$ when decreasing $\left.\theta\right)$ suggesting that the basal friction increases. In the inset of Fig. 12 is shown the $h_{\text {stop }}$ function to illustrate the possible link with $c_{\mu}$. We did not measure the basal friction directly and further investigations are needed on the influence of roughness. Whether this correlation between the function $h_{\text {stop }}$ and the fitting parameter $c_{\mu}$ is simply a coincidence or results from more fundamental physical phenomena remains an open question.

\subsection{Various contributions to the total force}

As illustrated in Fig. 13 and already mentioned in the paper, the typical time-varying force from the granular avalanche can be split into three phases: (i) a rapid, short-duration increase, (ii) a more or less pronounced force peak depending on the slope inclination, and (iii) a long-lasting force decrease (avalanche tail) until the avalanche comes to a standstill in the presence of a residual force. Figure 13 gives the model's predictions compared to the numerical data (using the calibration value of $c_{\mu}$ ) for the two extreme slopes $\theta=16^{\circ}$ and $\theta=32^{\circ}$, and for the intermediate slope $\theta=24^{\circ}$. Figure 13 also shows the different contributions to the total force: the dynamic force $F_{d}$, the hydrostatic force $F_{p}$, and the weight of the control volume $V_{0}$ minus the basal friction force $F_{w}-F_{f}$. The cross-comparison of the graphs given in Fig. 13 is highly informative on the flow-obstacle interaction.

First, during the avalanche phases (i) and (ii) previously mentioned, the distribution of the various contributions to the total force depends on the slope inclination. At high slope values (rapid-dilute regime), the dynamic contribution $F_{d}$ is dominant and largely contributes to the peak force: the contributions from $F_{p}$ and $F_{w}-F_{f}$ are insignificant. In this regime, the force is proportional to a purely dynamic force $1 / 2 \bar{\rho} \bar{u}^{2} h$. Here it is crucial to estimate the depth-averaged velocity and fluid density accurately to calculate the dynamic 
Physical Review E, Volume 82, Issue 4, 041302 (2010)

URL: http://link.aps.org/doi/10.1103/PhysRevE.82.041302

DOI: 10.1103/PhysRevE.82.041302

force. Accurate estimations of the deflecting angle $\alpha$ and the coefficient $\beta$ (related to the velocity profile in depth) and $\kappa$ (related to velocity reduction) are also of crucial importance to derive the so-called drag coefficient $2 \beta[1-(1-\kappa \alpha) \cos \alpha]$. At this stage, we only used empirical arguments to derive the deflecting angle $\alpha$ and the velocity reduction coefficient $\kappa$ [29]. At low slope values (a dense regime close to the quasi-static regime), the contribution of $F_{w}-F_{f}$ is dominant: the contribution $F_{p}$ is also important but to a lesser extent. The dynamic contribution is here largely inconsequential. In this regime, it is crucial to estimate the hydrostatic force (flow depth $h$, fluid density $\bar{\rho}$, and coefficient $k$ ) accurately as well as the properties of the control volume upstream of the obstacle (dead zone length $L$ and basal friction $\mu_{z m}$ also derived here from simple empirical arguments).

Second, during the avalanche phase (iii) previously mentioned (avalanche tail), the contribution to the total force mainly stems from $F_{w}-F_{f}$, whatever the slope inclination. Consequently, the conclusions drawn immediately above for the dense regime close to the quasi-static regime at low slope values are also valid here for any slope.

Third, the so-called granular liquid regime corresponds to a transition state between these two asymptotic behaviors for which all the contributions should be taken into account to determine the total time-varying force exerted on the wall by a dense granular avalanche. The hydrodynamic model presented in this paper is a good candidate to fulfill this requirement.

\section{CONCLUSION}

This paper has described a simple depth-averaged hydrodynamic model allowing one to derive the time-averaged force exerted by finite volume granular avalanches on a wall normal to the incident flow. Basic model equations were derived from a previous analytical model proposed for steady flow conditions [29]. The interaction of the granular stream with the wall is characterized by the formation of a dead zone upstream of the obstacle. This dead zone largely influences the resulting force on the wall. In the case of granular avalanches, we had to take into account the dead zone changes over time. This was described by an exponential variation in time. The proposed exponential law was shown to predict the dead zone length, roughly estimated from discrete numerical simulations, relatively well.

The hydrodynamic model predicted that the force was the sum of three contributions: (i) a dynamic force $F_{d}$, (ii) a hydrostatic force $F_{p}$, and (iii) the difference between the weight 
Physical Review E, Volume 82, Issue 4, 041302 (2010)

URL: http://link.aps.org/doi/10.1103/PhysRevE.82.041302

DOI: 10.1103/PhysRevE.82.041302

of the control volume (fluid volume disturbed by the wall) and its basal friction with the bottom, $F_{w}-F_{f}$. The first two forces were accurately determined. The third one largely influenced the total force (i) at low slope inclinations and (ii) the residual force for all slope values. It was shown to depend mainly on the value of the control volume (related to the dead zone length) and the basal friction coefficient. As the control volume was accurately estimated, the basal friction coefficient between the dead zone and the bottom remained to be evaluated. The model's prediction was first found to be in good agreement with the numerical values for most of the slopes in terms of the maximum force. The basal friction coefficient was then used as a fitting parameter. It was possible to accurately match the force decrease over time obtained from the discrete numerical simulations. The fitting process gave a basal friction coefficient depending on the slope. Above the critical maximum angle $\theta_{\max }$, no frictional force seems to be mobilized at the base. The wall is submitted to the dynamic and hydrostatic forces plus the weight of the control volume. Below $\theta_{\max }$ the frictional force sharply increases when the slope inclination decreases. The basal frictional force contributes to decreasing the force to which the wall is submitted. At this stage, we were not able to explain this peculiar behavior. We suggested relating it to the existence of the function $h_{\text {stop }}(\theta)$ evidenced from previous experimental and numerical simulations [27, 35, 37].

Today, advanced models based on depth-averaged equations initiated by the pioneering work of Savage and Hutter [39], recently revisited [38], are able to reproduce the dynamics of granular avalanches observed in small-scale experiments. With these models, it is not possible to compute the force exerted by the granular stream on a wall. The model proposed herein is encouraging in that it provides a simple analytical approach to derive the force if the flow features (flow depth, depth-averaged velocity, and density) are determined a priori by a depth-averaged model. Further research is needed to investigate the parameters derived from empirical arguments and needed to close the model. This paper has been restricted to a wall spanning the flow and consisting of a flat obstacle normal to the incident flow. Future investigations are needed on more complex flow geometries with possible 3D effects including lateral fluxes and other obstacles when the flow conditions correspond to the granular liquid regime for which stagnant zones can occur in the absence of a granular jump. 
Physical Review E, Volume 82, Issue 4, 041302 (2010)

URL: http://link.aps.org/doi/10.1103/PhysRevE.82.041302

DOI: 10.1103/PhysRevE.82.041302

\section{ACKNOWLEGMENTS}

This study received financial support from the French National Research Agency (ANRMONHA). The authors would like to thank to an anonymous referee for the constructive criticisms which helped to improve the quality of the paper.

[1] U. Tuzun, and R.M. Nedderman, Chem. Eng. Sci. 40, 337 (1985).

[2] R.M. Iverson, Reviews of Geophysics 35 (3), 245 (1997).

[3] Y.C. Tai, J.M.N.T. Gray, K. Hutter, and S. Noelle, Annal. Glac. 32, 281 (2001).

[4] B. Sovilla, M. Schaer, M. Kern, and P. Bartelt, J. Geophys. Res. 113, F01010 (2008).

[5] T. Faug, P. Gauer, K. Lied, M. Naaim, J. Geophys. Res. 113, F03009 (2008).

[6] X. Cui, J.M.N.T. Gray, and T. Johannesson, J. Geophys. Res. 112, F04012 (2007).

[7] K. Wieghardt, Mech. Res. Commun. 1, 3 (1974).

[8] K. Wieghardt, Ann. Rev. Fluid. Mech. 7, 89 (1975).

[9] R. Albert, M.A. Pfeifer, A.-L. Barabasi, and P. Schiffer, Phys. Rev. Lett. 82, 205 (1999).

[10] I. Albert, P. Tegzes, B. Kahng, R. Albert, J.G. Sample, M. Pfeifer, A.-L. Barabasi, T. Vicsek, and P. Schiffer, Phys. Rev. Lett. 84, 5122 (2000).

[11] I. Albert, J.G. Sample, A.J. Morss, S. Rajagopalan, A.-L. Barabasi, P. Schiffer, Phys. Rev. E 64, 061303 (2001).

[12] G. Hill, S. Yeung, and S.A. Koehler, Europhys. Lett. 72 (1), 137 (2005).

[13] C. Nouguier, C. Bohatier, J.J. Moreau, F. Radjai, Granul. Matter 2, 171 (2000).

[14] D. Chehata, R. Zenit, and C.R. Wassgren, Phys. Fluids 15 (6), 1622-1631 (2003).

[15] C.R. Wassgren, J.A. Cordova, R. Zenit, and A. Karion, Phys. Fluids 15 (11), 3318-3330 (2003).

[16] R. Bharadwaj, C. Wassgren, and R. Zenit, Phys. fluids 18 (4), 043301 (2006).

[17] V. Buchholtz and T. Poschel, Granular Matter 1 (1998).

[18] E.C. Rericha, C. Bizon, M.D. Shattuck, and H.L. Swinney, Phys. Rev. Lett. 88, 014302 (2001).

[19] A. Levy and M. Sayed, Phys. Fluids 19 (2), 023302 (2007).

[20] A. Levy and M. Sayed, Powder Tech. 181 (2), 137-148 (2008).

[21] J.M.N. Gray, Y.-C. Tai, and S. Noelle, J. Fluid Mech., 161 (2003). 
Physical Review E, Volume 82, Issue 4, 041302 (2010)

URL: http://link.aps.org/doi/10.1103/PhysRevE.82.041302

DOI: 10.1103/PhysRevE.82.041302

[22] M.-C. Chiou, Y. Wang, and K. Hutter, Acta Mech. 175 (14), 105 (2005).

[23] K.M. Hakonardottir and A.J. Hogg., Phys. Fluids 17, 077101 (2005).

[24] J.F. Boudet, Y. Amarouchene, B. Bonnier and H. Kellay, J. Fluid Mech. 572, 413 (2007).

[25] J.M.N.T. Gray, and X. Cui, J. Fluid Mech. 579, 113 (2007).

[26] H. Teufelsbauer, Y. Wang, M.-C. Chiou, and W. Wu, Granul. Matter 11, 209 (2009).

[27] Y. Forterre and O. Pouliquen, Ann. Rev. Fluid. Mech. 40, 1 (2008).

[28] T. Faug, P. Lachamp, and M. Naaim, Nat. Haz. Earth Syst. Sci. 2, 187 (2002).

[29] T. Faug, R. Beguin, and B. Chanut, Phys. Rev. E 80, 021305 (2009).

[30] C.E. Brennen, K. Sieck, and J. Paslaski, Powder Tech. 35, 31 (1983).

[31] S.B. Savage, J. Fluid Mech. 92, 53 (1979).

[32] S. P. Pudasaini, K. Hutter, S. S. Hsiau, S. C. Tai, Y. Wang, and R. Katzenbach, Phys. Fluids 19, $053302(2007)$.

[33] S. P. Pudasaini and C. Kroner, Phys. Rev. E 78, 041308 (2008).

[34] B. Chanut, T. Faug, and M. Naaim, AIP Conf. Proc. 1145, 609 (2009).

[35] O. Pouliquen, Phys. Fluids 11 (3), 542 (1999).

[36] L. E. Silbert, D. Ertas, G. S. Grest, T. C. Halsey, D. Levine, and S. J. Plimpton, Phys. Rev. E 64, 051302 (2001).

[37] GDR Midi, E. Phys. J.E 14, 341 (2004).

[38] O. Pouliquen and Y. Forterre, J. Fluid Mech. 453, 133-151 (2002).

[39] S.B. Savage and K. Hutter, J. Fluid Mech. 199, 177-215 (1989).

[40] P.A. Cundall and O.D.L. Strack, Geotechnique 29, 47 (1979).

[41] D. Ertas, G.S. Grest, T.C. Halsey, D. Levine, and L.E. Silbert, Europhys. Lett. 56 (2), 214 (2001).

[42] F. da Cruz F, S. Emam, M. Prochnow, J.N. Roux, and F. Chevoir, Phys. Rev. E 72, 021309 (2005).

[43] M. Prochnow, Ph.D. Thesis, Ecole Nationale des Ponts et Chaussées, Marne la vallée, France, 2002.

[44] D.J. Cumberland and R.J. Crawford, Elsevier, Amsterdam (1987).

[45] Y. Forterre and O. Pouliquen, J. Fluid Mech. 486, 21-50 (2003).

[46] O. Pouliquen, Phys. Fluids 11 (7), 1956 (1999). 

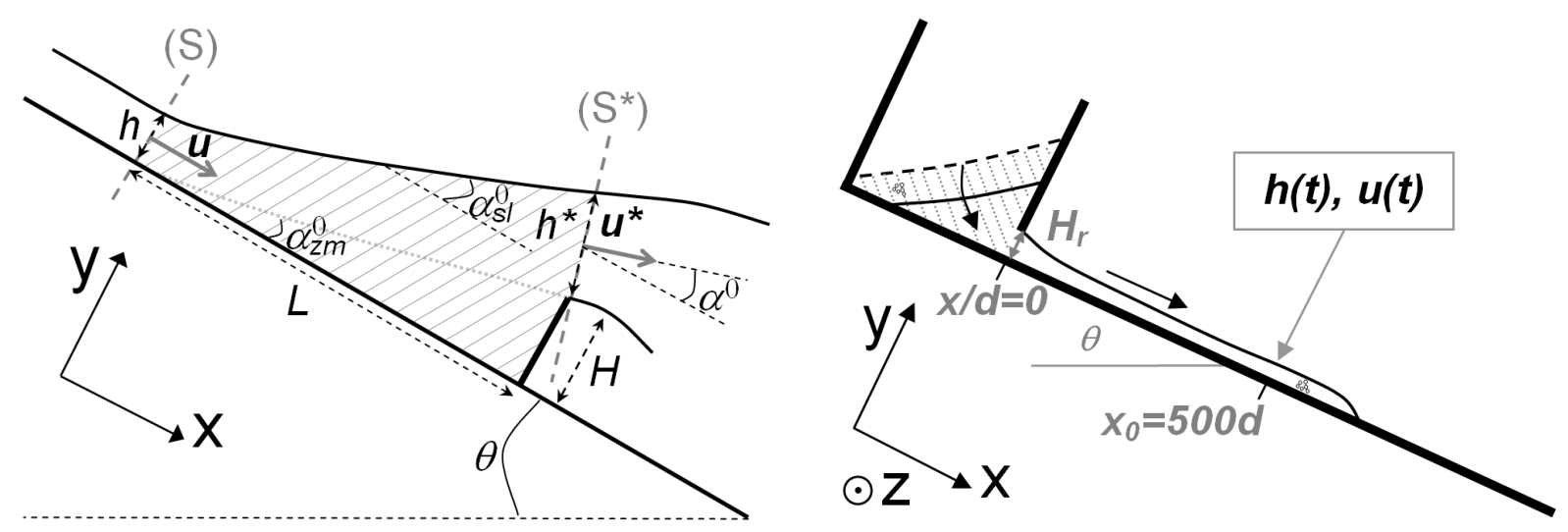

FIG. 1: (a) Sketch of the control volume $V_{0}$ (hatched zone) inside which momentum conservation is applied. $h$ and $\mathbf{u}$ are the flow depth and the mean velocity of the incoming flow at section $(S)$. The section $(S)$ is normal to the bottom and represents the beginning of the influence zone (length $L$ ) of the obstacle (height $H$ ). $L$ is the distance between the section $(S)$ and the foot of the obstacle. $h^{*}$ and $\mathbf{u}^{*}$ are the flow depth and the mean velocity of the flow at section $\left(S^{*}\right)$. Section $\left(S^{*}\right)$ is normal to the main direction of the outgoing flow of velocity $\mathbf{u}^{*}$. We defined the deflecting angle $\alpha$ between $\mathbf{u}^{*}$ and the bottom. $\theta$ is the bottom slope. Assuming a triangular shape for the dead zone, we also defined $\alpha_{z m}$ as the angle of the dead zone with the bottom and $\alpha_{s l}$ as the angle of the free-surface (inside $V_{0}$ ) with the bottom. (b) Sketch of the simulated system. A finite volume (hatched area) of an assembly of about 18000 spheres (mean diameter, $d$ ) is suddenly released from the reservoir (length $L_{r}=300 d$ ) by an aperture of height $H_{r}=35 d$, and the grains flow down the inclined slope. 


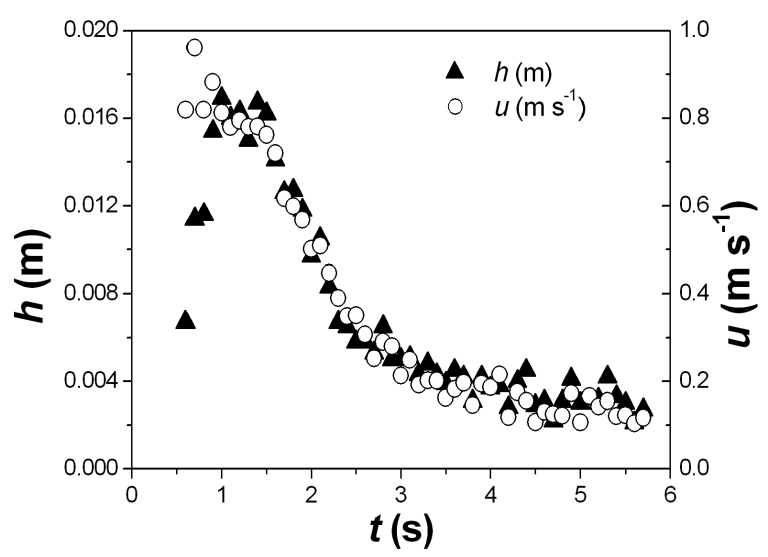

FIG. 2: Flow depth and velocity versus time measured at the position $x_{0} / d=500$ (see Fig 1b): example for $\theta=24^{\circ}$.

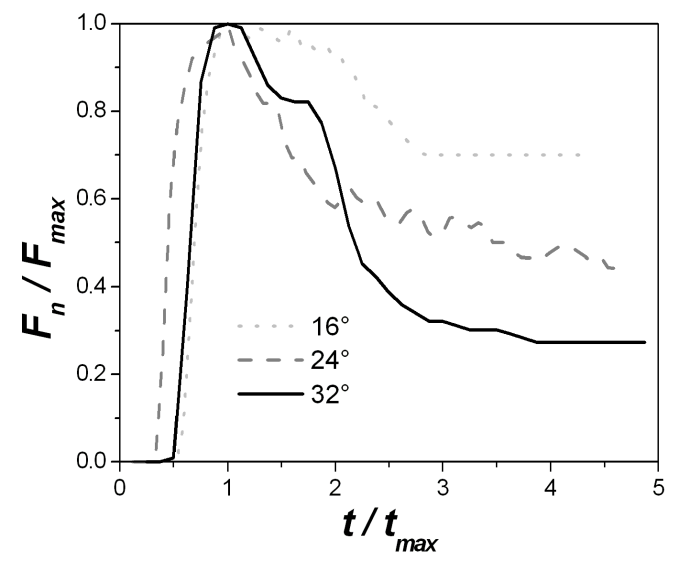

FIG. 3: Time-averaged normal force $F_{n}$ versus time (moving average over $0.2 \mathrm{~s}$ ). The results presented in this figure were obtained for $\theta=16^{\circ}, 24^{\circ}$ and $32^{\circ}$. The complete results are presented in [34] and in Sec. 3.3 when the numerical results and the hydrodynamic model prediction are cross-compared. 


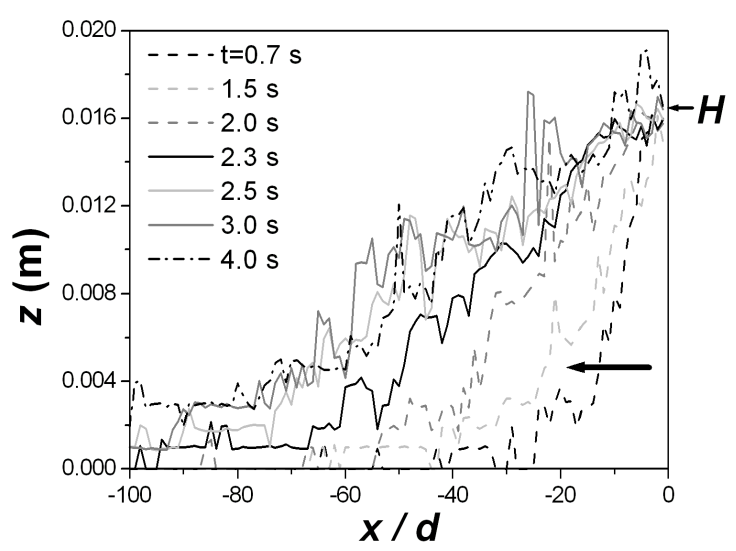

FIG. 4: Change over time of the dead zone shape: example for $\theta=28^{\circ}$. The arrow indicates the propagation of the dead zone upstream of the obstacle $(x / d<0)$.

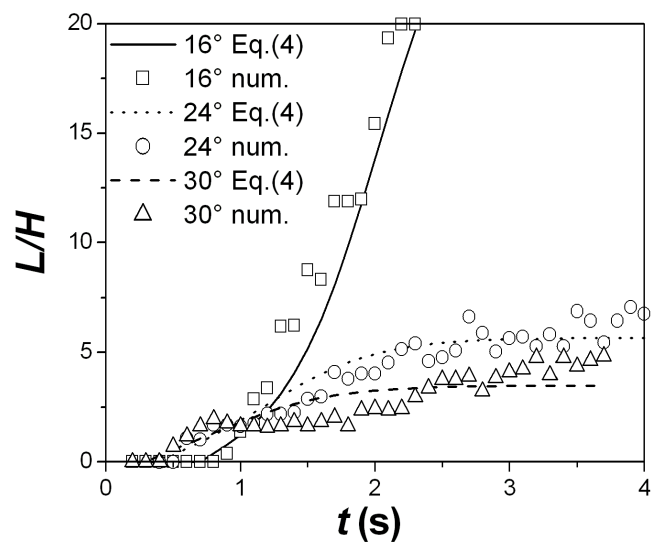

FIG. 5: Normalized dead zone length $L / H$ versus time $t(\mathrm{~s})$ : comparison between the numerical data and the predictions from Eq. (4) with $\tau=0.40 \mathrm{~s}$. Examples for $\theta=16^{\circ}, 24^{\circ}$ and $30^{\circ}$. 

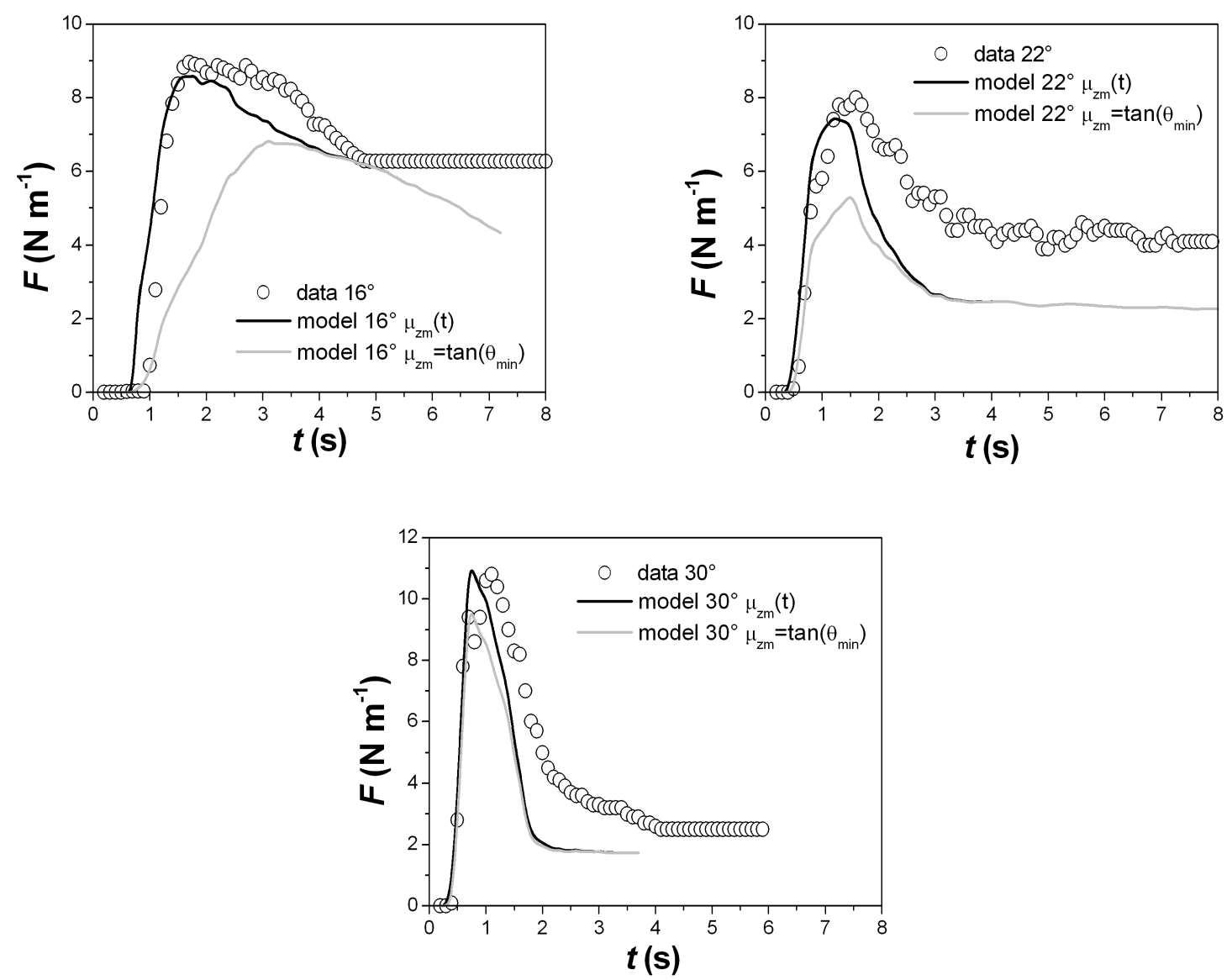

FIG. 6: Time-averaged force versus time for various slope inclinations: comparison between the numerical data (circles) and the model predictions using $\mu_{z m}(t)=\tan \left[\theta-\alpha_{z m}(t)\right]$ (black line) or $\mu_{z m}=\tan \theta_{\min }$ (gray line) with the following values: $\theta_{\min }=14^{\circ}, \theta_{\min }=24^{\circ}, k=1, \kappa=0.32$ $(e=0.5), \beta=1$, and $\phi_{\max }=0.64$. Examples for $\theta=16^{\circ}, 22^{\circ}$ and $30^{\circ}$. 


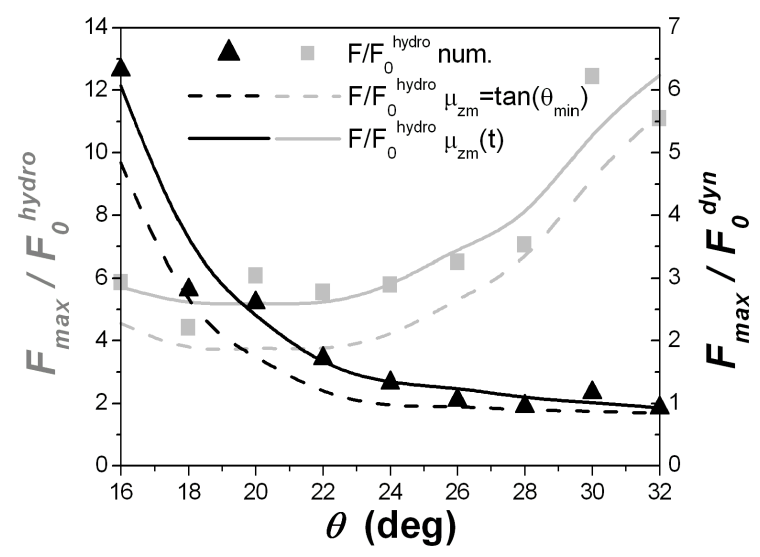

FIG. 7: Normalized maximum force $F_{\max } / F_{0}$ vs slope inclination $\theta$ with $F_{0}=F_{0}{ }^{\text {hydro }}$ (gray) or $F_{0}=F_{0}^{d y n}$ (black). Model predictions (curves) compared to discrete numerical data (points). Model predictions are given for two values of $\mu_{z m}: \mu_{z m}=\tan \theta_{\min }$ (dash line) and $\mu_{z m}(t)=$ $\tan \left[\theta-\alpha_{z m}(t)\right]$ (solid line). 

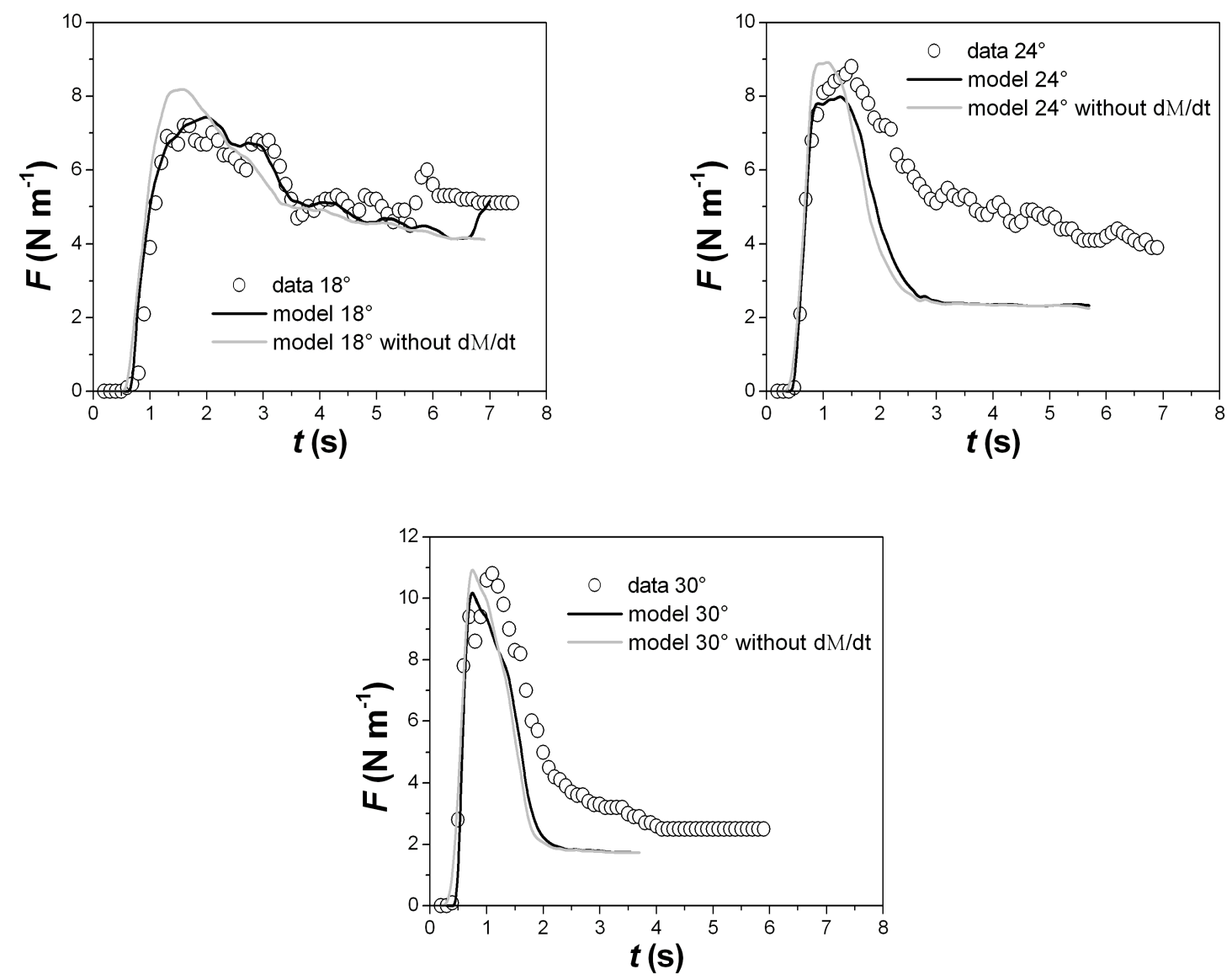

FIG. 8: Time averaged force versus time for various slope inclinations: comparison between the numerical data (circles) and the model's predictions including the time-derivative term in the momentum balance (black line) or not (gray line). The following values of the model's free parameters are used: $\theta_{\min }=14^{\circ}, \theta_{\min }=24^{\circ}, k=1, \kappa=0.32(e=0.5), \beta=1$, and $\phi_{\max }=0.64$. The friction is defined with respect to the time-varying geometry of the dead zone: $\mu_{z m}(t)=\tan \left[\theta-\alpha_{z m}(t)\right]$. Examples for $\theta=18^{\circ}, 24^{\circ}$ and $30^{\circ}$. 


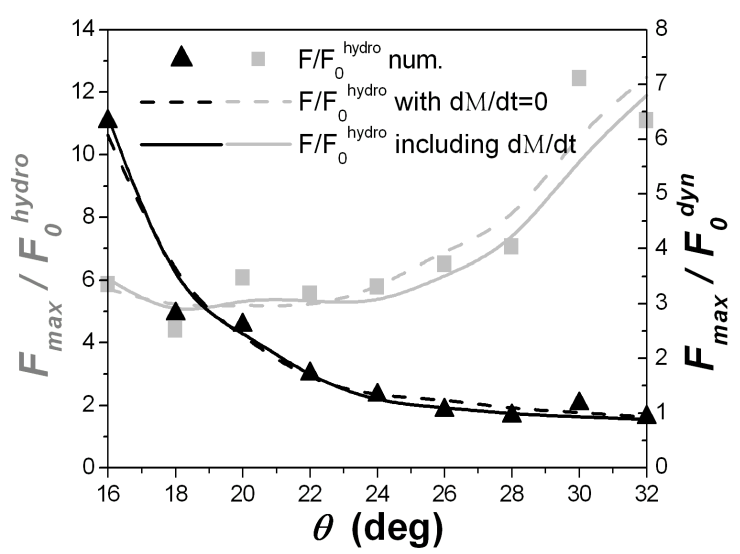

FIG. 9: Normalized maximum force $F_{\max } / F_{0}$ vs slope inclination $\theta$ with $F_{0}=F_{0}{ }^{\text {hydro }}$ (gray) or $F_{0}=F_{0}^{d y n}$ (black). Model predictions (curves) compared to discrete numerical data (points). The model predictions include the time-derivative term in the momentum balance (solid line) or not (dashed line). 

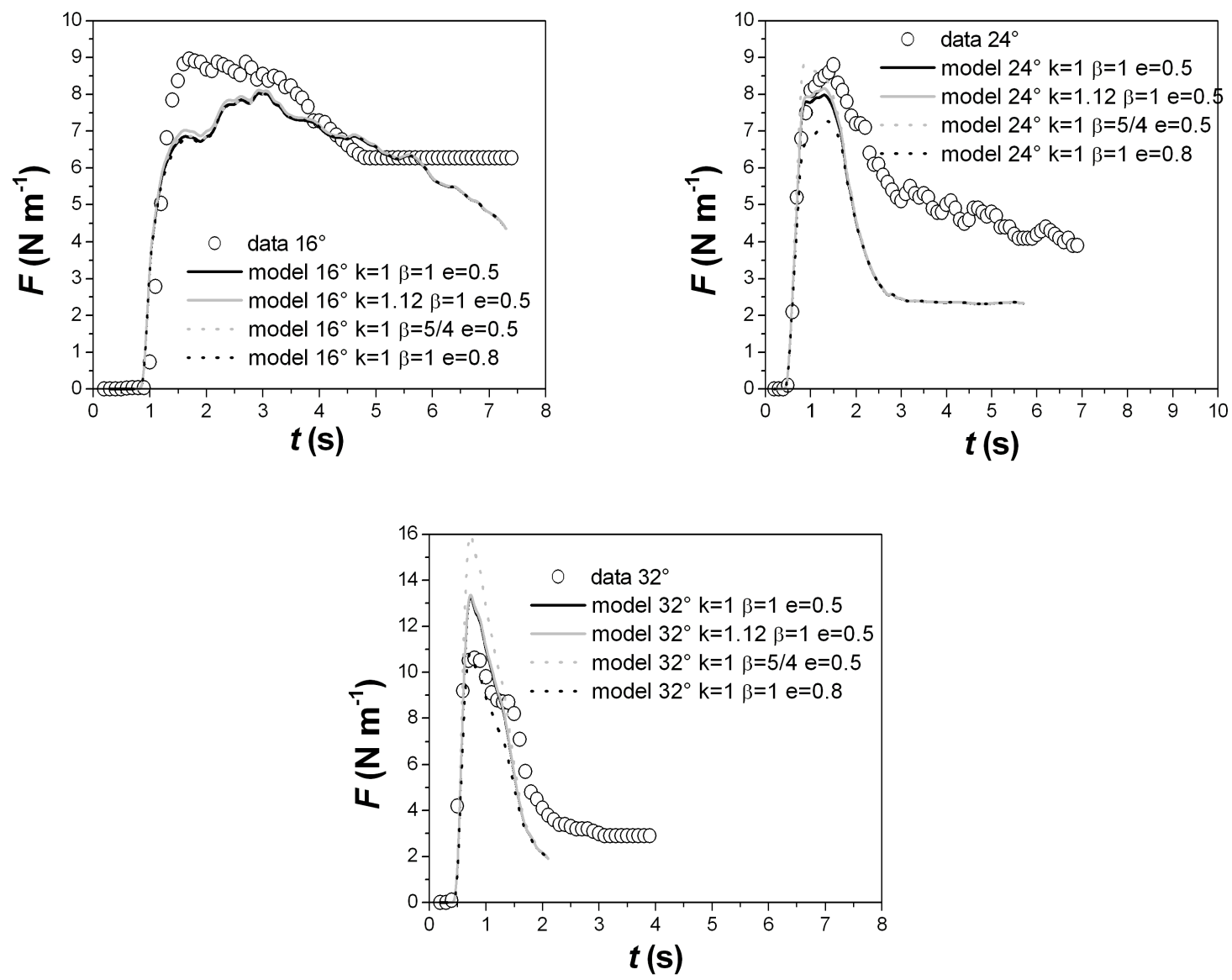

FIG. 10: Time-averaged force versus time: comparison between the numerical data (circles) and the model predictions using various sets of the model's free-parameters: (i) $k=1, \kappa=0.32(e=0.5)$ and $\beta=1$ (solid black line); (ii) $k=1.12, \kappa=0.32$ ( $e=0.5$ ), and $\beta=1$ (solid gray line); (iii) $k=1, \kappa=0.32(e=0.5)$, and $\beta=5 / 4$ (dashed gray line); (iv) $k=1, \kappa=0.13(e=0.8)$, and $\beta=1$ (dashed black line). The results are displayed for three slope inclinations: (a) $\theta=16^{\circ}$, (b) $24^{\circ}$, and (c) $32^{\circ}$. The model predictions are given using $\theta_{\min }=14^{\circ}, \theta_{\min }=24^{\circ}$, and $\mu_{z m}=\tan \left[\theta-\alpha_{z m}(t)\right]$. 

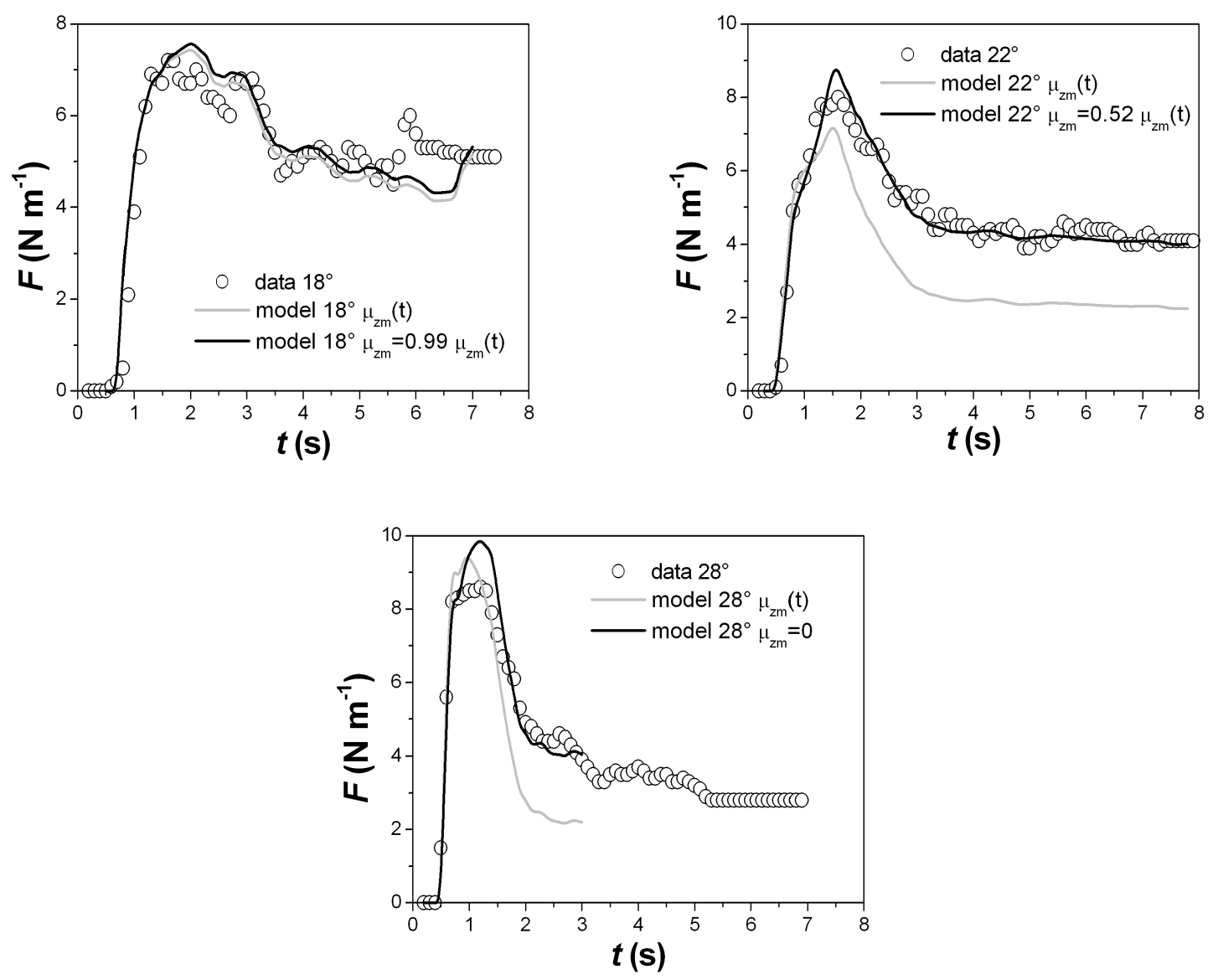

FIG. 11: Time-averaged force versus time for various slope inclinations: comparison between the numerical data (circles) and the model's predictions using $\mu_{z m}(t)=\tan \left[\theta-\alpha_{z m}(t)\right]$ (black line) or $\mu_{z m}^{*}(t)=c_{\mu} \tan \left[\theta-\alpha_{z m}(t)\right]$ (gray line) with the following values: $\theta_{\min }=14^{\circ}, \theta_{\min }=24^{\circ}, k=1$, $\kappa=0.32(e=0.5), \beta=1$ and $\phi_{\max }=0.64$. Examples for $\theta=18^{\circ}, 22^{\circ}$ and $28^{\circ}$. The best fit (least squares method) was obtained with: $c_{\mu}=0.99$ for $\theta=18^{\circ}, c_{\mu}=0.52$ for $22^{\circ}$ and $c_{\mu}=0$ (no basal friction force) for $\theta=28^{\circ}$. 


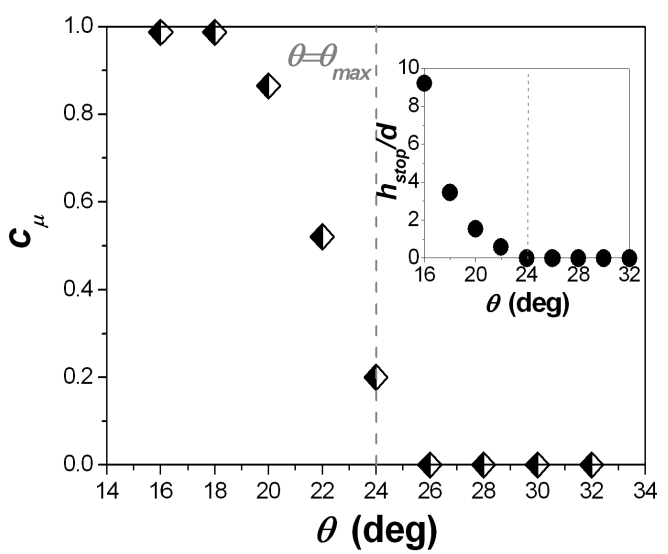

FIG. 12: Calibration coefficient $c_{\mu}$, defined by Eq. (7), versus the slope inclination $\theta$. The vertical dashed line marks the value of $\theta_{\max }$. Inset: function $h_{\text {stop }} / d$ versus $\theta$. 

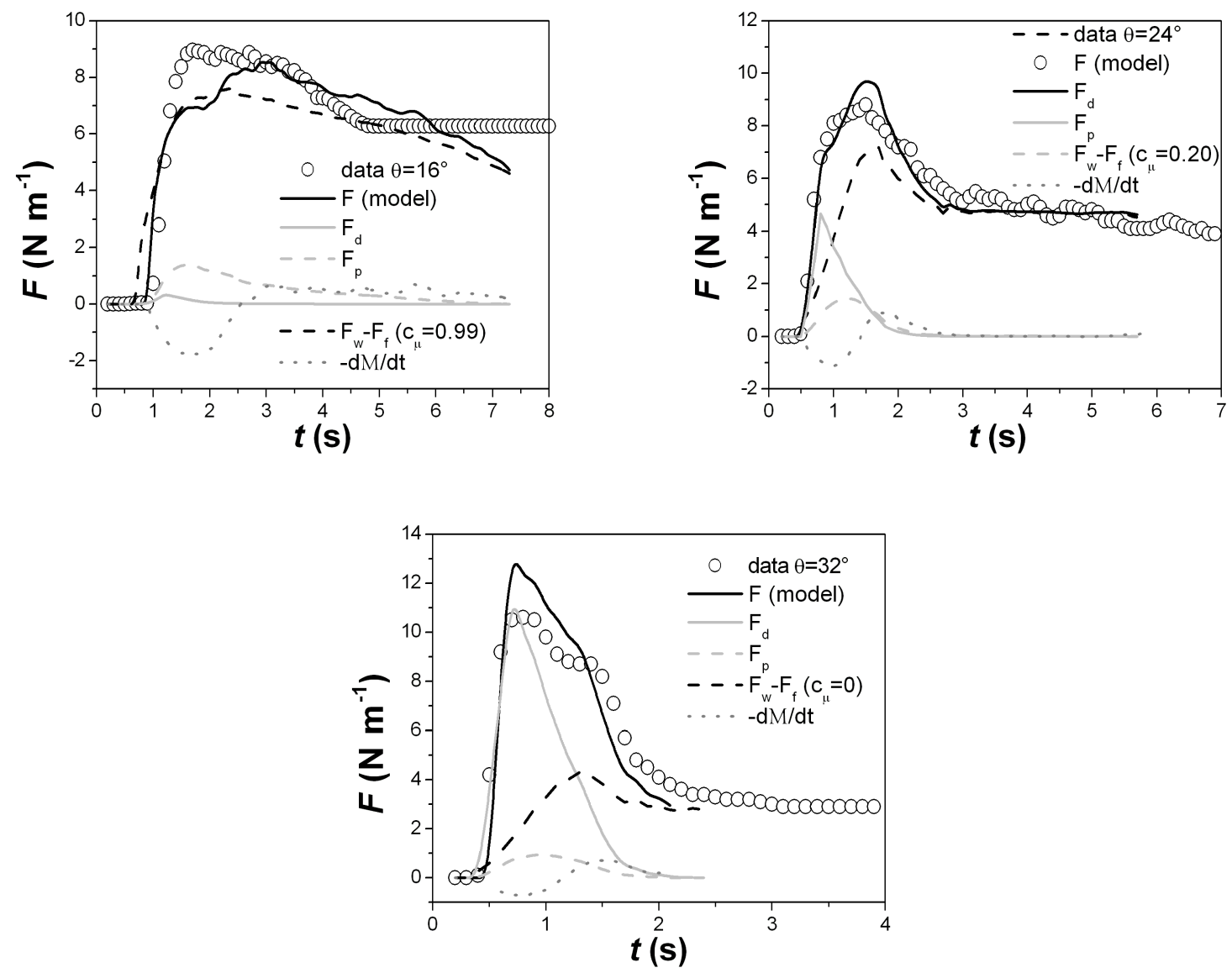

FIG. 13: Time-averaged force versus time: the model's predictions using the fitting coefficient $c_{\mu}$ (black line) compared to numerical data (circles), including the curves showing each contribution to the total force: $F_{d}$ (solid gray line), $F_{p}$ (dashed gray line) and $F_{w}-F_{f}$ (dashed black line). The results are presented for three slope inclinations: (a) $\theta=16^{\circ}$ (low slope), (b) $\theta=24^{\circ}$ (intermediate slope), and (c) $\theta=32^{\circ}$ (high slope). 